\title{
Synthesis of New Zirconium(IV) Complexes with Amino Acid Schiff Bases: Spectral, Molecular Modeling, and Fluorescence Studies
}

\author{
Har Lal Singh and Jangbhadur Singh \\ Department of Chemistry, Faculty of Engineering \& Technology, Mody Institute of Technology and Science, \\ Lakshmangarh, Sikar, Rajasthan 332311, India \\ Correspondence should be addressed to Har Lal Singh; hlsingh9@rediffmail.com
}

Received 29 September 2012; Accepted 5 January 2013

Academic Editor: Wei-Yin Sun

Copyright ( 2013 H. L. Singh and J. Singh. This is an open access article distributed under the Creative Commons Attribution License, which permits unrestricted use, distribution, and reproduction in any medium, provided the original work is properly cited.

\begin{abstract}
New zirconium(IV) complexes were synthesized with bidentate ligands and characterized by elemental analysis, molar conductance measurements, molecular weight determinations, IR, electronic, NMR $\left({ }^{1} \mathrm{H}\right.$ and $\left.{ }^{13} \mathrm{C}\right)$, fluorescence and molecular modeling studies. All the complexes are 1:2 electrolytes in nature and may be formulated as $\left[\mathrm{Zr}(\mathrm{L})_{2} \mathrm{Cl}_{2}\right]$ (where $\mathrm{L}$ is Schiff bases of amino acids and substituted isatin). The analytical data showed that the Schiff-base ligand acts as bidentate toward zirconium ion via the azomethine nitrogen and carboxylate oxygen. The conductivity values between $8.5-12.6 \Omega^{-1}, \mathrm{~mol}^{-1}, \mathrm{~cm}^{2}$ in DMF imply the presence of nonelectrolyte species. On the basis of spectral and molecular modeling studies, the resulting complexes are proposed to have octahedral geometries.
\end{abstract}

\section{Introduction}

The coordination chemistry of Schiff bases has been widely explored, though its use in supramolecular coordination chemistry remains largely unexplored. The Schiff-base moiety is potentially ambidentate and can coordinate through nitrogen with either oxygen or sulfur atoms. The vast literature on structural studies of Schiff-base complexes reveals some interesting features of their coordination behavior [16]. Schiff-base metal chelates have played a central role in the development of coordination chemistry. Metal complexes with Schiff-base ligands have been receiving considerable attention due to the pharmacological properties of both ligands and complexes [7-10]. Schiff-base derivatives exhibit a great variety of biological activities, such as antitumor $[11,12]$, antifungal [13, 14], antibacterial [15, 16], anticonvulsant [17] and antiviral [18] properties. The interest in the construction of Schiff-base coordination complexes by reacting transition metal ions with bidentate has been constantly growing over the past years [19-21]. Within this understanding lies an increased knowledge of molecular self-assembly, metalligand complexation, and disposition of metal binding sites.
By mastering these areas, new improved systems related to the fields of catalysis, supramolecular chemistry, and bioengineering can be achieved. Although the chemistry of zirconium complexes has been extensively studied, particularly in relation to their application as polymerization catalysts, the coordination chemistry of these oxophilic metals has concentrated on the use of oxygen donors. Many researchers have conducted on Schiff-base complexes; most of these complexes were found to be biologically active [22-25] as there has been considerable interest in the study of first-row transition metal Schiff-base complexes. However, relatively less work has appeared on the complexes of 2nd and 3rd rows transition metal ions. There is not much information on zirconium(IV) complexes from the available literature; therefore this paper reports the synthesis and characterization of zirconium(IV) complexes of Schiff bases derived from amino acids and isatins.

\section{Experimental}

2.1. Starting Materials. All chemicals used in the present work, $1 H$-indole-2,3-dione, 5-chloro- $1 H$-indole-2,3-dione, 
TABLE 1: Various Bond Lengths of Compound $\left[\mathrm{Zr}\left(\mathrm{L}^{4}\right)_{2} \mathrm{Cl}_{2}\right]$.

\begin{tabular}{|c|c|c|c|}
\hline S. No. & Atoms & Actual $(\AA)$ & Optimal $(\AA)$ \\
\hline 1 & $\mathrm{Zr}(4)-\mathrm{N}(3)$ & 2.1381 & \\
\hline 2 & $\mathrm{C}(41)-\mathrm{H}(66)$ & 1.1131 & 1.113 \\
\hline 3 & $\mathrm{C}(41)-\mathrm{H}(65)$ & 1.1126 & 1.113 \\
\hline 4 & $\mathrm{C}(39)-\mathrm{H}(64)$ & 1.1137 & 1.113 \\
\hline 5 & $\mathrm{C}(39)-\mathrm{H}(63)$ & 1.1153 & 1.113 \\
\hline 6 & $\mathrm{C}(38)-\mathrm{H}(62)$ & 1.1125 & 1.113 \\
\hline 7 & $\mathrm{C}(38)-\mathrm{H}(61)$ & 1.1135 & 1.113 \\
\hline 8 & $\mathrm{C}(38)-\mathrm{H}(60)$ & 1.1127 & 1.113 \\
\hline 9 & $\mathrm{C}(36)-\mathrm{H}(59)$ & 1.1132 & 1.113 \\
\hline 10 & $\mathrm{C}(36)-\mathrm{H}(58)$ & 1.1141 & 1.113 \\
\hline 11 & $\mathrm{C}(35)-\mathrm{H}(57)$ & 1.1142 & 1.113 \\
\hline 12 & $\mathrm{C}(35)-\mathrm{H}(56)$ & 1.1132 & 1.113 \\
\hline 13 & $\mathrm{C}(34)-\mathrm{H}(55)$ & 1.0939 & 1.1 \\
\hline 14 & $\mathrm{C}(33)-\mathrm{H}(54)$ & 1.1023 & 1.1 \\
\hline 15 & $\mathrm{C}(32)-\mathrm{H}(53)$ & 1.1023 & 1.1 \\
\hline 16 & $\mathrm{C}(31)-\mathrm{H}(52)$ & 1.1016 & 1.1 \\
\hline 17 & $\mathrm{~N}(27)-\mathrm{H}(51)$ & 1.0444 & 1.05 \\
\hline 18 & $\mathrm{C}(25)-\mathrm{H}(50)$ & 1.1159 & 1.113 \\
\hline 19 & $\mathrm{C}(25)-\mathrm{H}(49)$ & 1.1146 & 1.113 \\
\hline 20 & $\mathrm{C}(24)-\mathrm{H}(48)$ & 1.1012 & 1.1 \\
\hline 21 & $\mathrm{C}(23)-\mathrm{H}(47)$ & 1.1061 & 1.1 \\
\hline 22 & $\mathrm{C}(22)-\mathrm{H}(46)$ & 1.1049 & 1.1 \\
\hline 23 & $\mathrm{C}(21)-\mathrm{H}(45)$ & 1.1012 & 1.1 \\
\hline 24 & $\mathrm{~N}(17)-\mathrm{H}(44)$ & 1.0446 & 1.05 \\
\hline 25 & $\mathrm{C}(8)-\mathrm{H}(43)$ & 1.1138 & 1.113 \\
\hline 26 & $\mathrm{C}(2)-\mathrm{H}(42)$ & 1.1165 & 1.113 \\
\hline 27 & $S(40)-C(41)$ & 1.8167 & 1.815 \\
\hline 28 & $\mathrm{C}(39)-\mathrm{S}(40)$ & 1.8236 & 1.815 \\
\hline 29 & C(25)-C(39) & 1.5388 & 1.523 \\
\hline 30 & $\mathrm{~S}(37)-\mathrm{C}(38)$ & 1.8142 & 1.815 \\
\hline 31 & $C(36)-S(37)$ & 1.8255 & 1.815 \\
\hline 32 & $\mathrm{C}(35)-\mathrm{C}(36)$ & 1.5393 & 1.523 \\
\hline 33 & $\mathrm{~N}(9)-\operatorname{Zr}(4)$ & 2.1345 & \\
\hline 34 & $\operatorname{Zr}(4)-\mathrm{O}(5)$ & 2.0916 & \\
\hline 35 & $C(2)-C(35)$ & 1.5486 & 1.523 \\
\hline 36 & $\mathrm{C}(34)-\mathrm{C}(29)$ & 1.4014 & 1.42 \\
\hline 37 & $\mathrm{C}(33)-\mathrm{C}(34)$ & 1.3978 & 1.42 \\
\hline 38 & $\mathrm{C}(32)-\mathrm{C}(33)$ & 1.3994 & 1.42 \\
\hline 39 & $C(31)-C(32)$ & 1.3929 & 1.42 \\
\hline 40 & $\mathrm{C}(28)-\mathrm{C}(31)$ & 1.3971 & 1.42 \\
\hline 41 & $\mathrm{C}(26)-\mathrm{O}(30)$ & 1.2293 & 1.208 \\
\hline 42 & C(29)-C(15) & 1.4774 & 1.503 \\
\hline 43 & $\mathrm{C}(28)-\mathrm{C}(29)$ & 1.4077 & 1.42 \\
\hline 44 & $\mathrm{~N}(27)-\mathrm{C}(28)$ & 1.3928 & 1.462 \\
\hline 45 & $\mathrm{C}(26)-\mathrm{N}(27)$ & 1.3678 & 1.462 \\
\hline 46 & $\mathrm{C}(15)-\mathrm{C}(26)$ & 1.5141 & 1.517 \\
\hline 47 & $\mathrm{C}(8)-\mathrm{C}(25)$ & 1.5398 & 1.523 \\
\hline 48 & $\mathrm{C}(24)-\mathrm{C}(19)$ & 1.4832 & 1.42 \\
\hline 49 & $\mathrm{C}(23)-\mathrm{C}(24)$ & 1.5134 & 1.42 \\
\hline 50 & $C(22)-C(23)$ & 1.5149 & 1.42 \\
\hline 51 & $C(21)-C(22)$ & 1.5092 & 1.42 \\
\hline
\end{tabular}

TABLE 1: Continued.

\begin{tabular}{lccc}
\hline S. No. & Atoms & Actual $(\AA)$ & Optimal $(\AA)$ \\
\hline 52 & $\mathrm{C}(18)-\mathrm{C}(21)$ & 1.4845 & 1.42 \\
53 & $\mathrm{C}(16)-\mathrm{O}(20)$ & 1.3098 & 1.208 \\
54 & $\mathrm{C}(19)-\mathrm{C}(14)$ & 1.5193 & 1.503 \\
55 & $\mathrm{C}(18)-\mathrm{C}(19)$ & 1.374 & 1.42 \\
56 & $\mathrm{~N}(17)-\mathrm{C}(18)$ & 1.3476 & 1.462 \\
57 & $\mathrm{C}(16)-\mathrm{N}(17)$ & 1.3903 & 1.462 \\
58 & $\mathrm{C}(14)-\mathrm{C}(16)$ & 1.5503 & 1.517 \\
59 & $\mathrm{~N}(3)-\mathrm{C}(15)$ & 1.2988 & 1.26 \\
60 & $\mathrm{~N}(9)-\mathrm{C}(14)$ & 2.1882 & 1.26 \\
61 & $\mathrm{C}(1)-\mathrm{O}(13)$ & 1.2142 & 1.208 \\
62 & $\mathrm{C}(7)-\mathrm{O}(12)$ & 1.2117 & 1.208 \\
63 & $\mathrm{Zr}(4)-\mathrm{Cl}(11)$ & 2.4423 & \\
64 & $\mathrm{Zr}(4)-\mathrm{Cl}(10)$ & 2.4453 & \\
65 & $\mathrm{C}(8)-\mathrm{N}(9)$ & 1.4968 & 1.47 \\
66 & $\mathrm{C}(7)-\mathrm{C}(8)$ & 1.5279 & 1.509 \\
67 & $\mathrm{O}(6)-\mathrm{C}(7)$ & 1.3594 & 1.338 \\
68 & $\mathrm{C}(41)-\mathrm{H}(67)$ & 1.1129 & 1.113 \\
69 & $\mathrm{O}(5)-\mathrm{C}(1)$ & 1.3551 & 1.338 \\
70 & $\mathrm{Zr}(4)-\mathrm{O}(6)$ & 2.0914 & \\
71 & $\mathrm{C}(2)-\mathrm{N}(3)$ & 1.5032 & 1.47 \\
72 & $\mathrm{C}(1)-\mathrm{C}(2)$ & 1.5292 & \\
\hline
\end{tabular}

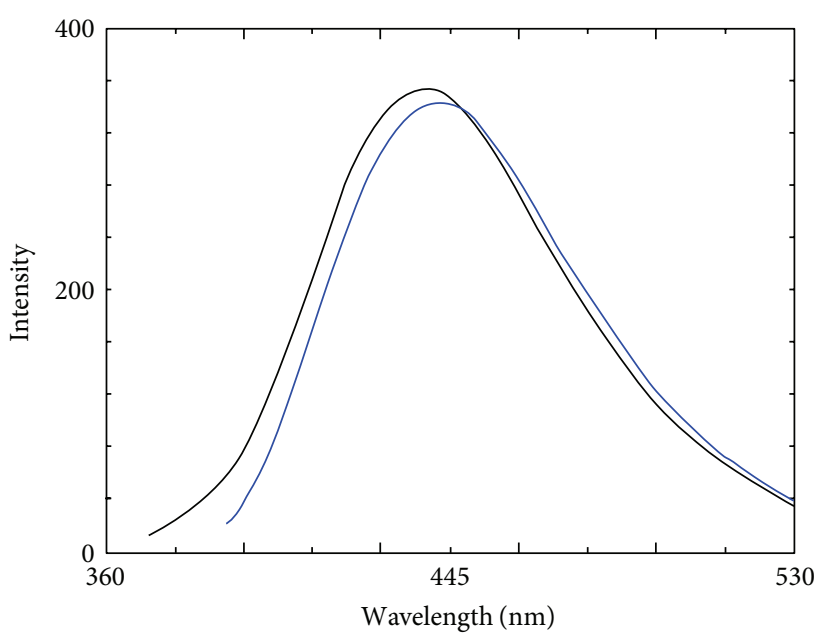

Figure 1: Emission spectra of the ligand $\left(\mathrm{L}^{3} \mathrm{H}\right)$.

amino acids (glycine, alanine, valine, methionine, phenylalanine, and tryptophan), and oxozirconium(IV) chloride, were of analytical grade. The ligands were prepared by the condensation of isatins with amino acids as described earlier $[9,26]$.

2.2. Analytical Procedures. Solvents used were dried and purified by standard methods and moisture was excluded from the glass apparatus using $\mathrm{CaCl}_{2}$ drying tubes. The melting points of the compounds were determined on a capillary melting point apparatus and were not corrected. The purity of the compounds was confirmed by thin layer chromatography using silica gel-G glass plates as the stationary phase and 
TABLE 2: Various Bond Angles of Compound $\left[\mathrm{Zr}\left(\mathrm{L}^{4}\right)_{2} \mathrm{Cl}_{2}\right]$.

\begin{tabular}{|c|c|c|c|}
\hline S. No. & Atoms & Actual $\left({ }^{\circ}\right)$ & Optimal $\left(^{\circ}\right)$ \\
\hline 1 & $\mathrm{H}(67)-\mathrm{C}(41)-\mathrm{H}(66)$ & 109.418 & 109 \\
\hline 2 & $\mathrm{H}(67)-\mathrm{C}(41)-\mathrm{H}(65)$ & 108.9614 & 109 \\
\hline 3 & $\mathrm{H}(67)-\mathrm{C}(41)-\mathrm{S}(40)$ & 110.3566 & 109.3 \\
\hline 4 & $\mathrm{H}(66)-\mathrm{C}(41)-\mathrm{H}(65)$ & 108.7912 & 109 \\
\hline 5 & $\mathrm{H}(66)-\mathrm{C}(41)-\mathrm{S}(40)$ & 109.9408 & 109.3 \\
\hline 6 & $\mathrm{H}(65)-\mathrm{C}(41)-\mathrm{S}(40)$ & 109.3437 & 109.3 \\
\hline 7 & $C(41)-S(40)-C(39)$ & 97.5564 & 96.3 \\
\hline 8 & $\mathrm{H}(64)-\mathrm{C}(39)-\mathrm{H}(63)$ & 107.5686 & 109.4 \\
\hline 9 & $\mathrm{H}(64)-\mathrm{C}(39)-\mathrm{S}(40)$ & 110.1883 & 112 \\
\hline 10 & $\mathrm{H}(64)-\mathrm{C}(39)-\mathrm{C}(25)$ & 110.4229 & 109.41 \\
\hline 11 & $\mathrm{H}(63)-\mathrm{C}(39)-\mathrm{S}(40)$ & 109.8119 & 112 \\
\hline 12 & $\mathrm{H}(63)-\mathrm{C}(39)-\mathrm{C}(25)$ & 109.1207 & 109.41 \\
\hline 13 & $\mathrm{~S}(40)-\mathrm{C}(39)-\mathrm{C}(25)$ & 109.6938 & 106.5 \\
\hline 14 & $\mathrm{H}(62)-\mathrm{C}(38)-\mathrm{H}(61)$ & 109.6623 & 109 \\
\hline 15 & $\mathrm{H}(62)-\mathrm{C}(38)-\mathrm{H}(60)$ & 109.0937 & 109 \\
\hline 16 & $\mathrm{H}(62)-\mathrm{C}(38)-\mathrm{S}(37)$ & 110.3571 & 109.3 \\
\hline 17 & $\mathrm{H}(61)-\mathrm{C}(38)-\mathrm{H}(60)$ & 108.6879 & 109 \\
\hline 18 & $\mathrm{H}(61)-\mathrm{C}(38)-\mathrm{S}(37)$ & 110.0128 & 109.3 \\
\hline 19 & $\mathrm{H}(60)-\mathrm{C}(38)-\mathrm{S}(37)$ & 108.9946 & 109.3 \\
\hline 20 & $C(38)-S(37)-C(36)$ & 98.533 & 96.3 \\
\hline 21 & $\mathrm{H}(59)-\mathrm{C}(36)-\mathrm{H}(58)$ & 107.1198 & 109.4 \\
\hline 22 & $\mathrm{H}(59)-\mathrm{C}(36)-\mathrm{S}(37)$ & 110.417 & 112 \\
\hline 23 & $\mathrm{H}(59)-\mathrm{C}(36)-\mathrm{C}(35)$ & 109.5515 & 109.41 \\
\hline 24 & $\mathrm{H}(58)-\mathrm{C}(36)-\mathrm{S}(37)$ & 110.7645 & 112 \\
\hline 25 & $\mathrm{H}(58)-\mathrm{C}(36)-\mathrm{C}(35)$ & 110.8347 & 109.41 \\
\hline 26 & $S(37)-C(36)-C(35)$ & 108.1586 & 106.5 \\
\hline 27 & $\mathrm{H}(57)-\mathrm{C}(35)-\mathrm{H}(56)$ & 104.9845 & 109.4 \\
\hline 28 & $\mathrm{H}(57)-\mathrm{C}(35)-\mathrm{C}(36)$ & 108.6578 & 109.41 \\
\hline 29 & $\mathrm{H}(57)-\mathrm{C}(35)-\mathrm{C}(2)$ & 110.8409 & 109.41 \\
\hline 30 & $\mathrm{H}(56)-\mathrm{C}(35)-\mathrm{C}(36)$ & 108.9072 & 109.41 \\
\hline 31 & $\mathrm{H}(56)-\mathrm{C}(35)-\mathrm{C}(2)$ & 109.5018 & 109.41 \\
\hline 32 & $C(36)-C(35)-C(2)$ & 113.5779 & 109.5 \\
\hline 33 & $\mathrm{H}(55)-\mathrm{C}(34)-\mathrm{C}(29)$ & 122.3033 & 120 \\
\hline 34 & $\mathrm{H}(55)-\mathrm{C}(34)-\mathrm{C}(33)$ & 117.5306 & 120 \\
\hline 35 & $\mathrm{C}(29)-\mathrm{C}(34)-\mathrm{C}(33)$ & 120.1661 & \\
\hline 36 & $\mathrm{H}(54)-\mathrm{C}(33)-\mathrm{C}(34)$ & 119.6447 & 120 \\
\hline 37 & $\mathrm{H}(54)-\mathrm{C}(33)-\mathrm{C}(32)$ & 119.4129 & 120 \\
\hline 38 & $\mathrm{C}(34)-\mathrm{C}(33)-\mathrm{C}(32)$ & 120.9424 & \\
\hline 39 & $\mathrm{H}(53)-\mathrm{C}(32)-\mathrm{C}(33)$ & 119.7786 & 120 \\
\hline 40 & $\mathrm{H}(53)-\mathrm{C}(32)-\mathrm{C}(31)$ & 120.1954 & 120 \\
\hline 41 & $\mathrm{C}(33)-\mathrm{C}(32)-\mathrm{C}(31)$ & 119.9868 & \\
\hline 42 & $\mathrm{H}(52)-\mathrm{C}(31)-\mathrm{C}(32)$ & 121.1904 & 120 \\
\hline 43 & $\mathrm{H}(52)-\mathrm{C}(31)-\mathrm{C}(28)$ & 120.678 & 120 \\
\hline 44 & $C(32)-C(31)-C(28)$ & 118.1313 & \\
\hline 45 & $\mathrm{C}(34)-\mathrm{C}(29)-\mathrm{C}(15)$ & 134.3781 & 120 \\
\hline 46 & $\mathrm{C}(34)-\mathrm{C}(29)-\mathrm{C}(28)$ & 117.1629 & 120 \\
\hline 47 & $C(15)-C(29)-C(28)$ & 108.4549 & 120 \\
\hline 48 & $\mathrm{C}(31)-\mathrm{C}(28)-\mathrm{C}(29)$ & 123.0239 & 120 \\
\hline 49 & $\mathrm{C}(31)-\mathrm{C}(28)-\mathrm{N}(27)$ & 126.8775 & 120 \\
\hline 50 & $\mathrm{C}(29)-\mathrm{C}(28)-\mathrm{N}(27)$ & 110.0936 & 120 \\
\hline 51 & $\mathrm{H}(51)-\mathrm{N}(27)-\mathrm{C}(28)$ & 126.0741 & 118 \\
\hline
\end{tabular}

TABLE 2: Continued.

\begin{tabular}{|c|c|c|c|}
\hline S. No. & Atoms & Actual $\left({ }^{\circ}\right)$ & Optimal $\left(^{\circ}\right)$ \\
\hline 52 & $\mathrm{H}(51)-\mathrm{N}(27)-\mathrm{C}(26)$ & 125.3088 & 118 \\
\hline 53 & $\mathrm{C}(28)-\mathrm{N}(27)-\mathrm{C}(26)$ & 108.5425 & \\
\hline 54 & $\mathrm{O}(30)-\mathrm{C}(26)-\mathrm{N}(27)$ & 120.8581 & 122.6 \\
\hline 55 & $\mathrm{O}(30)-\mathrm{C}(26)-\mathrm{C}(15)$ & 129.2063 & 123 \\
\hline 56 & $\mathrm{~N}(27)-\mathrm{C}(26)-\mathrm{C}(15)$ & 109.9356 & 122 \\
\hline 57 & $\mathrm{H}(50)-\mathrm{C}(25)-\mathrm{H}(49)$ & 106.678 & 109.4 \\
\hline 58 & $\mathrm{H}(50)-\mathrm{C}(25)-\mathrm{C}(39)$ & 108.7135 & 109.41 \\
\hline 59 & $\mathrm{H}(50)-\mathrm{C}(25)-\mathrm{C}(8)$ & 108.393 & 109.41 \\
\hline 60 & $\mathrm{H}(49)-\mathrm{C}(25)-\mathrm{C}(39)$ & 111.2794 & 109.41 \\
\hline 61 & $\mathrm{H}(49)-\mathrm{C}(25)-\mathrm{C}(8)$ & 109.454 & 109.41 \\
\hline 62 & $\mathrm{C}(39)-\mathrm{C}(25)-\mathrm{C}(8)$ & 112.1296 & 109.5 \\
\hline 63 & $\mathrm{H}(48)-\mathrm{C}(24)-\mathrm{C}(19)$ & 117.3341 & 120 \\
\hline 64 & $\mathrm{H}(48)-\mathrm{C}(24)-\mathrm{C}(23)$ & 117.009 & 120 \\
\hline 65 & $\mathrm{C}(19)-\mathrm{C}(24)-\mathrm{C}(23)$ & 119.9311 & \\
\hline 66 & $\mathrm{H}(47)-\mathrm{C}(23)-\mathrm{C}(24)$ & 113.9434 & 120 \\
\hline 67 & $\mathrm{H}(47)-\mathrm{C}(23)-\mathrm{C}(22)$ & 114.0848 & 120 \\
\hline 68 & $\mathrm{C}(24)-\mathrm{C}(23)-\mathrm{C}(22)$ & 118.3693 & \\
\hline 69 & $\mathrm{H}(46)-\mathrm{C}(22)-\mathrm{C}(23)$ & 115.8207 & 120 \\
\hline 70 & $\mathrm{H}(46)-\mathrm{C}(22)-\mathrm{C}(21)$ & 115.0649 & 120 \\
\hline 71 & $C(23)-C(22)-C(21)$ & 118.1479 & \\
\hline 72 & $\mathrm{H}(45)-\mathrm{C}(21)-\mathrm{C}(22)$ & 119.4891 & 120 \\
\hline 73 & $\mathrm{H}(45)-\mathrm{C}(21)-\mathrm{C}(18)$ & 118.9569 & 120 \\
\hline 74 & $C(22)-C(21)-C(18)$ & 118.0658 & \\
\hline 75 & $C(24)-C(19)-C(14)$ & 124.1732 & 120 \\
\hline 76 & $\mathrm{C}(24)-\mathrm{C}(19)-\mathrm{C}(18)$ & 120.434 & 120 \\
\hline 77 & $\mathrm{C}(14)-\mathrm{C}(19)-\mathrm{C}(18)$ & 114.4563 & 120 \\
\hline 78 & $\mathrm{C}(21)-\mathrm{C}(18)-\mathrm{C}(19)$ & 123.9331 & 120 \\
\hline 79 & $\mathrm{C}(21)-\mathrm{C}(18)-\mathrm{N}(17)$ & 126.1831 & 120 \\
\hline 80 & $\mathrm{C}(19)-\mathrm{C}(18)-\mathrm{N}(17)$ & 109.7946 & 120 \\
\hline 81 & $\mathrm{H}(44)-\mathrm{N}(17)-\mathrm{C}(18)$ & 126.029 & 118 \\
\hline 82 & $\mathrm{H}(44)-\mathrm{N}(17)-\mathrm{C}(16)$ & 126.0518 & 118 \\
\hline 83 & $\mathrm{C}(18)-\mathrm{N}(17)-\mathrm{C}(16)$ & 107.8705 & \\
\hline 84 & $\mathrm{O}(20)-\mathrm{C}(16)-\mathrm{N}(17)$ & 122.4042 & 122.6 \\
\hline 85 & $\mathrm{O}(20)-\mathrm{C}(16)-\mathrm{C}(14)$ & 123.6108 & 123 \\
\hline 86 & $\mathrm{~N}(17)-\mathrm{C}(16)-\mathrm{C}(14)$ & 113.7879 & 122 \\
\hline 87 & $C(29)-C(15)-C(26)$ & 101.2183 & 117.6 \\
\hline 88 & $\mathrm{C}(29)-\mathrm{C}(15)-\mathrm{N}(3)$ & 129.5068 & 120 \\
\hline 89 & $C(26)-C(15)-N(3)$ & 129.128 & 120 \\
\hline 90 & $C(19)-C(14)-C(16)$ & 93.7144 & 117.6 \\
\hline 91 & $\mathrm{C}(19)-\mathrm{C}(14)-\mathrm{N}(9)$ & 101.1826 & 120 \\
\hline 92 & $\mathrm{C}(16)-\mathrm{C}(14)-\mathrm{N}(9)$ & 103.5499 & 120 \\
\hline 93 & $\mathrm{Zr}(4)-\mathrm{N}(9)-\mathrm{C}(14)$ & 104.5285 & \\
\hline 94 & $\operatorname{Zr}(4)-\mathrm{N}(9)-\mathrm{C}(8)$ & 111.1129 & \\
\hline 95 & $\mathrm{C}(14)-\mathrm{N}(9)-\mathrm{C}(8)$ & 111.1476 & \\
\hline 96 & $\mathrm{H}(43)-\mathrm{C}(8)-\mathrm{C}(25)$ & 108.1727 & 109.39 \\
\hline 97 & $\mathrm{H}(43)-\mathrm{C}(8)-\mathrm{N}(9)$ & 109.7641 & 107.5 \\
\hline 98 & $\mathrm{H}(43)-\mathrm{C}(8)-\mathrm{C}(7)$ & 106.4941 & 107.9 \\
\hline 99 & $\mathrm{C}(25)-\mathrm{C}(8)-\mathrm{N}(9)$ & 111.9987 & \\
\hline 100 & $\mathrm{C}(25)-\mathrm{C}(8)-\mathrm{C}(7)$ & 113.8847 & 109.9 \\
\hline 101 & $\mathrm{~N}(9)-\mathrm{C}(8)-\mathrm{C}(7)$ & 106.3523 & \\
\hline 102 & $\mathrm{O}(12)-\mathrm{C}(7)-\mathrm{C}(8)$ & 125.0648 & 122.5 \\
\hline
\end{tabular}


TABLE 2: Continued.

\begin{tabular}{|c|c|c|c|}
\hline S. No. & Atoms & Actual $\left({ }^{\circ}\right)$ & Optimal $\left({ }^{\circ}\right)$ \\
\hline 103 & $\mathrm{O}(12)-\mathrm{C}(7)-\mathrm{O}(6)$ & 124.6366 & 122 \\
\hline 104 & $\mathrm{C}(8)-\mathrm{C}(7)-\mathrm{O}(6)$ & 110.2827 & 107.1 \\
\hline 105 & $\mathrm{Zr}(4)-\mathrm{O}(6)-\mathrm{C}(7)$ & 111.3282 & \\
\hline 106 & $\mathrm{Zr}(4)-\mathrm{O}(5)-\mathrm{C}(1)$ & 114.0377 & \\
\hline 107 & $\mathrm{~N}(3)-\mathrm{Zr}(4)-\mathrm{O}(5)$ & 80.1135 & \\
\hline 108 & $\mathrm{~N}(3)-\mathrm{Zr}(4)-\mathrm{O}(6)$ & 128.6218 & \\
\hline 109 & $\mathrm{~N}(3)-\mathrm{Zr}(4)-\mathrm{N}(9)$ & 149.0865 & \\
\hline 110 & $\mathrm{~N}(3)-\mathrm{Zr}(4)-\mathrm{Cl}(11)$ & 88.0663 & \\
\hline 111 & $\mathrm{~N}(3)-\mathrm{Zr}(4)-\mathrm{Cl}(10)$ & 84.2757 & \\
\hline 112 & $\mathrm{O}(5)-\mathrm{Zr}(4)-\mathrm{O}(6)$ & 148.2304 & \\
\hline 113 & $\mathrm{O}(5)-\mathrm{Zr}(4)-\mathrm{N}(9)$ & 78.2701 & \\
\hline 114 & $\mathrm{O}(5)-\mathrm{Zr}(4)-\mathrm{Cl}(11)$ & 85.1672 & \\
\hline 115 & $\mathrm{O}(5)-\mathrm{Zr}(4)-\mathrm{Cl}(10)$ & 116.7647 & \\
\hline 116 & $\mathrm{O}(6)-\mathrm{Zr}(4)-\mathrm{N}(9)$ & 78.9572 & \\
\hline 117 & $\mathrm{O}(6)-\mathrm{Zr}(4)-\mathrm{Cl}(11)$ & 82.9908 & \\
\hline 118 & $\mathrm{O}(6)-\mathrm{Zr}(4)-\mathrm{Cl}(10)$ & 83.2655 & \\
\hline 119 & $\mathrm{~N}(9)-\operatorname{Zr}(4)-\mathrm{Cl}(11)$ & 111.6987 & \\
\hline 120 & $\mathrm{~N}(9)-\mathrm{Zr}(4)-\mathrm{Cl}(10)$ & 86.0092 & \\
\hline 121 & $\mathrm{Cl}(11)-\mathrm{Zr}(4)-\mathrm{Cl}(10)$ & 154.9219 & \\
\hline 122 & $\mathrm{Zr}(4)-\mathrm{N}(3)-\mathrm{C}(15)$ & 121.0647 & \\
\hline 123 & $\operatorname{Zr}(4)-N(3)-C(2)$ & 107.937 & \\
\hline 124 & $C(15)-N(3)-C(2)$ & 120.234 & \\
\hline 125 & $\mathrm{H}(42)-\mathrm{C}(2)-\mathrm{C}(35)$ & 110.432 & 109.39 \\
\hline 126 & $\mathrm{H}(42)-\mathrm{C}(2)-\mathrm{N}(3)$ & 106.5807 & 107.5 \\
\hline 127 & $\mathrm{H}(42)-\mathrm{C}(2)-\mathrm{C}(1)$ & 102.9891 & 107.9 \\
\hline 128 & $\mathrm{C}(35)-\mathrm{C}(2)-\mathrm{N}(3)$ & 118.1743 & \\
\hline 129 & $C(35)-C(2)-C(1)$ & 109.8863 & 109.9 \\
\hline 130 & $\mathrm{~N}(3)-\mathrm{C}(2)-\mathrm{C}(1)$ & 107.6576 & \\
\hline 131 & $\mathrm{O}(13)-\mathrm{C}(1)-\mathrm{O}(5)$ & 124.3628 & 122 \\
\hline 132 & $\mathrm{O}(13)-\mathrm{C}(1)-\mathrm{C}(2)$ & 123.8243 & 122.5 \\
\hline 133 & $\mathrm{O}(5)-\mathrm{C}(1)-\mathrm{C}(2)$ & 111.734 & 107.1 \\
\hline
\end{tabular}

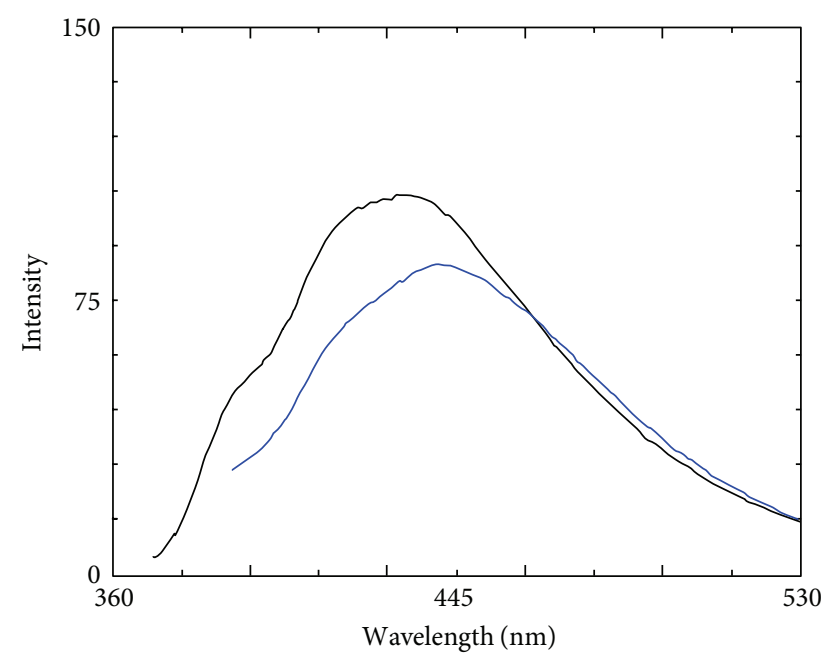

FIgURE 2: Emission spectra of the $\mathrm{Zr}\left(\mathrm{L}^{3}\right)_{2} \mathrm{Cl}_{2}$ complex. benzene and ethanol $(9: 1)$ as the mobile phase. Zirconium was determined gravimetrically as its oxide, $\mathrm{ZrO}_{2}$. Nitrogen and sulfur were determined by Kjeldahl's and Messenger's methods, respectively. Molecular weight determinations were carried out by the Rast camphor method.

2.3. Physical Measurements. The IR spectra of samples in $\mathrm{KBr}$ pellets were recorded on an FTIR spectrophotometer model SP-2, Perkin Elmer in the range of $4000-400 \mathrm{~cm}^{-1}$. The electronic spectra of the ligands and their metal complexes were recorded in dry DMSO, on a thermo-, double-beam spectrophotometer UV 1 , in the range of $800-200 \mathrm{~nm}$. The fluorescence studies of Schiff base and its metal complexes were recorded on Shimadzu RF-5301PC spectrophotometer. The molar conductance of the complexes was measured on $10^{-3} \mathrm{M}$ DMF solutions using Systronics conductivity bride model 305. ${ }^{1} \mathrm{H}$ and ${ }^{13} \mathrm{C}$ NMR spectra were recorded on Bruker avance II $(400 \mathrm{MHz})$ FTNMR spectrometer at the SAIF, Punjab University, Chandigarh, using DMSO- $\mathrm{d}_{6}$ as the solvent and tetramethylsilane (TMS) as an internal standard.

2.4. Molecular Modeling Studies. The molecular modeling of a representative compound is carried out on a CS Chem 3D ultramolecular modeling and analysis programme, interactive graphics programme that enables rapid structure building and geometry optimization with minimum energy and molecular display.

2.5. Preparation of Zirconium Complexes. The complexes were prepared by treating oxozirconium(IV) chloride $(1.61 \mathrm{mmol})$ in methanol with the corresponding Schiff bases $(3.22 \mathrm{mmol})$ in the same solvent. The mixture was refluxed for three hours on a water bath after which the crystals of the complex separate out on cooling; the mixture was concentrated on a steam bath until about one-third of the solution remained. The concentrated solution was cooled after which the crystals were filtered, washed with methanol, and then dried in vacuum at $45 \pm 5^{\circ} \mathrm{C}$ after repeated washing with dry cyclohexane. The compounds were purified by recrystallization from the same solvent. The purity of the compounds was checked by TLC using silica gel G as adsorbent.

Compound $\mathrm{Zr}\left(\mathrm{L}^{1}\right)_{2} \mathrm{Cl}_{2}$ was prepared by reacting zirconium(IV) dichloride with ligand $\left(\mathrm{L}^{1} \mathrm{H}\right)$; colour, red; yield, $0.218 \mathrm{~g} ; \mathrm{mp}, 170^{\circ} \mathrm{C}(\mathrm{d})$ and elemental analysis (\%), calcd. for $\mathrm{C}_{20} \mathrm{H}_{14} \mathrm{Cl}_{2} \mathrm{~N}_{4} \mathrm{O}_{6} \mathrm{Zr}$ : C, 42.26; H, 2.48; N, 9.86; found, C: 42.33; H, 2.57; N, 9.81; molecular weight: found, 580.12, calcd. 568.48. Molar conductance (DMF, $10^{-3}, \Omega^{-1}, \mathrm{moL}^{-1}, \mathrm{~cm}^{2}$ ): $10.8 ;{ }^{1} \mathrm{H}$ NMR (DMSO-d 6 , $\delta$ ppm, $400 \mathrm{MHz}$ ): $4.28(\mathrm{~s}, 2 \mathrm{H}$, $\left.\mathrm{N}-\mathrm{CH}_{2}-\right), 8.06(\mathrm{~s}, 1 \mathrm{H}, \mathrm{NH}), 7.18-7.78(\mathrm{~m}, 4 \mathrm{H}$, aromatic); ${ }^{13} \mathrm{C}$ NMR (DMSO, $\left.\delta \mathrm{ppm}\right): 186.3$ (COO), $52.1\left(-\mathrm{CH}_{2}-\right)$, $153.8(\mathrm{C}=\mathrm{N}), 167.3(\mathrm{C}=\mathrm{O}), 148.65,131.2,129.9,125.3,122.7$, 119.5 (aromatic carbons); UV-visible $(\lambda \max , \mathrm{nm}): 220,260$, 320 , 370; infrared $\left(\mathrm{KBr}, \mathrm{cm}^{-1}\right): v(\mathrm{C}=\mathrm{N}), 1610 ; v(\mathrm{C}=\mathrm{O}), 1722$; $v_{\text {asym }}(\mathrm{COO}), 1595 ; v_{\text {sym }}(\mathrm{COO}), 1325 ; v(\mathrm{Zr} \leftarrow \mathrm{N}), 535 ; v(\mathrm{Zr}-$ O), 465 .

Compound $\mathrm{Zr}\left(\mathrm{L}^{2}\right)_{2} \mathrm{Cl}_{2}$ was prepared by reacting zirconium(IV) dichloride with ligand $\left(\mathrm{L}^{2} \mathrm{H}\right)$; colour, reddish 
brown; yield, $0.317 \mathrm{~g}$; mp, $200^{\circ} \mathrm{C}(\mathrm{d})$ and elemental analysis (\%), calcd. for $\mathrm{C}_{22} \mathrm{H}_{18} \mathrm{Cl}_{2} \mathrm{~N}_{4} \mathrm{O}_{6} \mathrm{Zr}$ : C, 44.30; $\mathrm{H}, 3.04 ; \mathrm{N}$, 9.39; found, $\mathrm{C}$ : 44.46; H, 3.10; N, 9.33; molecular weight: found, 590.68, calcd. 596.53. Molar conductance (DMF, $10^{-3}, \Omega^{-1}$, $\mathrm{moL}^{-1}, \mathrm{~cm}^{2}$ ): $11.5 ;{ }^{1} \mathrm{H}$ NMR (DMSO-d $\left.\mathrm{d}_{6}, \delta \mathrm{ppm}, 400 \mathrm{MHz}\right)$ : 4.70 (q, 1H, N-CH-); 8.32 (s, 1H, NH); 1.50 (d, 3H, C- $\mathrm{CH}_{3}$ ); 7.00-7.86 (m, 4H, aromatic); ${ }^{13} \mathrm{C} \mathrm{NMR} \mathrm{(DMSO,} \delta$ ppm): 180.7 (COO), 63.6 (-CH-), 150.2 (C=N), 159.6 (C=O), 1502.7, 138.3, $133.6,131.7,130.5,129.3,124.9,121.1$ (aromatic carbons); UVvisible $(\lambda \max , \mathrm{nm}): 220,260,320,370$; infrared $\left(\mathrm{KBr}, \mathrm{cm}^{-1}\right)$ : $\nu(\mathrm{C}=\mathrm{N}), 1617 ; \nu(\mathrm{C}=\mathrm{O}), 1728 ; v_{\text {asym }}(\mathrm{COO}), 1585 ; v_{\text {sym }}(\mathrm{COO})$, $1322 ; v(\mathrm{Zr} \leftarrow \mathrm{N}), 537 ; v(\mathrm{Zr}-\mathrm{O}), 460$.

Compound $\mathrm{Zr}\left(\mathrm{L}^{3}\right)_{2} \mathrm{Cl}_{2}$ was prepared by reacting zirconium(IV) dichloride with ligand $\left(\mathrm{L}^{3} \mathrm{H}\right)$; colour, brown; yield, $0.179 \mathrm{~g} ; \mathrm{mp}, 144^{\circ} \mathrm{C}(\mathrm{d})$ and elemental analysis (\%), calcd. for $\mathrm{C}_{26} \mathrm{H}_{26} \mathrm{Cl}_{2} \mathrm{~N}_{4} \mathrm{O}_{6} \mathrm{Zr}$ : C, 47.85; H, 4.02; N, 8.58; found, C: 47.89; $\mathrm{H}, 4.14 ; \mathrm{N}, 8.54$; molecular weight: found, 640.96, calcd. 652.64. Molar conductance (DMF, $10^{-3}, \Omega^{-1}, \mathrm{moL}^{-1}, \mathrm{~cm}^{2}$ ): $8.5 ;{ }^{1} \mathrm{H}$ NMR (DMSO-d ${ }_{6}, \delta$ ppm, $\left.400 \mathrm{MHz}\right): 4.26(\mathrm{~d}, 1 \mathrm{H}, \mathrm{N}-$ $\mathrm{CH}-) ; 8.40$ (s, 1H, NH); 2.16-2.30 (m, 1H, -CH-); 1.22 (d, $\left.3 \mathrm{H},-\mathrm{CH}_{3}\right) 7.11-7.65$ (m, $4 \mathrm{H}$, aromatic); ${ }^{13} \mathrm{C}$ NMR (DMSO, $\delta$ ppm): 183.1 (COO), 67.5 (-CH-), $18.4\left(-\mathrm{CH}_{3}\right), 152.5(\mathrm{C}=\mathrm{N})$, $161.2(\mathrm{C}=\mathrm{O}), 150.68,140.7,132.85,131.5,131.3,129.4,123.7$, 121.2 (aromatic carbons); UV-visible $(\lambda \max , \mathrm{nm}): 220,260$, 320, 370; infrared $\left(\mathrm{KBr}, \mathrm{cm}^{-1}\right): v(\mathrm{C}=\mathrm{N}), 1616 ; v(\mathrm{C}=\mathrm{O}), 1725$; $v_{\text {asym }}(\mathrm{COO}), 1588 ; v_{\text {sym }}(\mathrm{COO}), 1322 ;(\mathrm{Zr} \leftarrow \mathrm{N}), 530 ; v(\mathrm{Zr}-$ O), 462 .

Compound $\mathrm{Zr}\left(\mathrm{L}^{4}\right)_{2} \mathrm{Cl}_{2}$ was prepared by reacting zirconium(IV) dichloride with ligand $\left(\mathrm{L}^{4} \mathrm{H}\right)$; colour, reddish brown; yield, $0.120 \mathrm{~g} ; \mathrm{mp}, 160^{\circ} \mathrm{C}(\mathrm{d})$ and elemental analysis (\%), calcd. for $\mathrm{C}_{26} \mathrm{H}_{26} \mathrm{Cl}_{2} \mathrm{~N}_{4} \mathrm{O}_{6} \mathrm{~S}_{2} \mathrm{Zr}$ : C, 43.57; $\mathrm{H}, 3.66$; N, 7.82; found, $\mathrm{C}$ : 43.15; $\mathrm{H}, 3.70 ; \mathrm{N}, 7.70$; molecular weight: found, 723.11, calcd. 716.77. Molar conductance (DMF, $10^{-3}, \Omega^{-1}$, $\mathrm{moL}^{-1}, \mathrm{~cm}^{2}$ ): $12.2{ }^{1} \mathrm{H}$ NMR (DMSO-d $6, \delta \mathrm{ppm}, 400 \mathrm{MHz}$ ): $4.62(\mathrm{t}, 1 \mathrm{H}, \mathrm{N}-\mathrm{CH}-), 2.20-2.30\left(\mathrm{~m}, 4 \mathrm{H},-\mathrm{CH}_{2}-\right), 1.56(\mathrm{~s}, 3 \mathrm{H},-$ $\left.\mathrm{CH}_{3}\right), 8.00$ (s, $\left.1 \mathrm{H}, \mathrm{NH}\right), 7.10-7.72\left(\mathrm{~m}, 4 \mathrm{H}\right.$, aromatic); ${ }^{13} \mathrm{C} \mathrm{NMR}$ (DMSO, $\delta$ ppm): $185.6(\mathrm{COO}) ; 62.3(\mathrm{CH}) ; 26.8,30.2\left(\mathrm{CH}_{2}\right)$; $17.9\left(\mathrm{CH}_{3}\right) ; 153.7(\mathrm{C}=\mathrm{N}) ; 158.1(\mathrm{C}=\mathrm{O}) ; 144.86,136.2,132.5$, $128.24,122.6,117.3$ (aromatic carbons); UV-visible ( $\lambda \max$, $\mathrm{nm}): 218,260,378$; infrared $\left(\mathrm{KBr}, \mathrm{cm}^{-1}\right): v(\mathrm{C}=\mathrm{N}), 1608$; $v(\mathrm{C}=\mathrm{O}), 1722, v(\mathrm{NH}), 3130, v_{\text {asym }}(\mathrm{COO}), 1582 ; v_{\text {sym }}(\mathrm{COO})$, 1310; $v(\mathrm{Zr} \leftarrow \mathrm{N}), 542 ; v(\mathrm{Zr}-\mathrm{O}), 468$.

Compound $\mathrm{Zr}\left(\mathrm{L}^{5}\right)_{2} \mathrm{Cl}_{2}$ was prepared by reacting zirconium(IV) dichloride with ligand $\left(\mathrm{L}^{5} \mathrm{H}\right)$; colour, brown; yield, $0.194 \mathrm{~g} ; \mathrm{mp}, 254^{\circ} \mathrm{C}(\mathrm{d})$ and elemental analysis (\%), calcd. for $\mathrm{C}_{22} \mathrm{H}_{16} \mathrm{Cl}_{4} \mathrm{~N}_{4} \mathrm{O}_{6} \mathrm{Zr}$ : C, 39.71; $\mathrm{H}, 2.42 ; \mathrm{N}, 8.42$; found, $\mathrm{C}$ : $39.56 ; \mathrm{H}, 2.40 ; \mathrm{N}, 8.34$; molecular weight: found, 660.29, calcd. 665.42. Molar conductance (DMF, $10^{-3}, \Omega^{-1}, \mathrm{moL}^{-1}$, $\mathrm{cm}^{2}$ ): $11.9 ;{ }^{1} \mathrm{H}$ NMR (DMSO-d $6, \delta$ ppm, $400 \mathrm{MHz}$ ): 4.15 (q, $1 \mathrm{H}, \mathrm{N}-\mathrm{CH}-$ ); 1.40 (d, 3H, $-\mathrm{CH}_{3}$ ); 8.00 (s, $\left.1 \mathrm{H}, \mathrm{NH}\right), 7.02-$ $7.60(\mathrm{~m}, 3 \mathrm{H}$, aromatic); UV-visible $(\lambda \max , \mathrm{nm}): 220,260$, 320, 370; infrared $\left(\mathrm{KBr}, \mathrm{cm}^{-1}\right): v(\mathrm{C}=\mathrm{N}), 1612 ; v(\mathrm{C}=\mathrm{O}), 1720$; $v_{\text {asym }}(\mathrm{COO}), 1585 ; v_{\text {sym }}(\mathrm{COO}), 1315 ; v(\mathrm{Zr} \leftarrow \mathrm{N}), 530 ; v(\mathrm{Zr}-$ O), 460 .

Compound $\mathrm{Zr}\left(\mathrm{L}^{6}\right)_{2} \mathrm{Cl}_{2}$ was prepared by reacting zirconium(IV) dichloride with ligand $\left(\mathrm{L}^{6} \mathrm{H}\right)$; colour, brown; yield, $0.203 \mathrm{~g} ; \mathrm{mp}, 180^{\circ} \mathrm{C}(\mathrm{d})$ and elemental analysis (\%), calcd. for $\mathrm{C}_{26} \mathrm{H}_{24} \mathrm{Cl}_{4} \mathrm{~N}_{4} \mathrm{O}_{6} \mathrm{Zr}$ : C, 43.28; $\mathrm{H}, 3.35$; N, 7.77; found, C:
42.97; H, 3.41; N, 7.62; molecular weight: found, 708.58, calcd. 721.53. Molar conductance (DMF, $10^{-3}, \Omega^{-1}, \mathrm{moL}^{-1}, \mathrm{~cm}^{2}$ ): 10.6; ${ }^{1} \mathrm{H}$ NMR (DMSO-d 6 , $\delta$ ppm, $400 \mathrm{MHz}$ ): $4.00(\mathrm{t}, 1 \mathrm{H}$, $\mathrm{N}-\mathrm{CH}-$ ); $2.25-2.28\left(\mathrm{~m}, 1 \mathrm{H},-\mathrm{CH}_{2}-\right)$; 8.40 (s, 1H, NH), 7.01$7.70(\mathrm{~m}, 3 \mathrm{H}$, aromatic); UV-visible $(\lambda \max , \mathrm{nm}): 220,260$, 320, 370; infrared $\left(\mathrm{KBr}, \mathrm{cm}^{-1}\right): v(\mathrm{C}=\mathrm{N}), 1615 ; v(\mathrm{C}=\mathrm{O}), 1722$; $v_{\text {asym }}(\mathrm{COO}), 1590 ; v_{\text {sym }}(\mathrm{COO}), 1320 ; v(\mathrm{Zr} \leftarrow \mathrm{N}), 538 ; v(\mathrm{Zr}-$ O), 466.

Compound $\mathrm{Zr}\left(\mathrm{L}^{7}\right)_{2} \mathrm{Cl}_{2}$ was prepared by reacting zirconium(IV) dichloride with ligand $\left(\mathrm{L}^{7} \mathrm{H}\right)$; colour, brown; yield, $0.332 \mathrm{~g} ; \mathrm{mp}, 210^{\circ} \mathrm{C}(\mathrm{d})$ and elemental analysis (\%) calcd. for $\mathrm{C}_{34} \mathrm{H}_{26} \mathrm{Cl}_{2} \mathrm{~N}_{4} \mathrm{O}_{6} \mathrm{Zr}$ : C, 54.54; $\mathrm{H}, 3.50 ; \mathrm{N}, 7.48$; found, C: $54.44 ; \mathrm{H}, 3.55 ; \mathrm{N}, 7.54$; molecular weight: found, 755.64, calcd. 748.72. Molar conductance (DMF, $10^{-3}, \Omega^{-1}, \mathrm{moL}^{-1}$, $\mathrm{cm}^{2}$ ): $12.6 ;{ }^{1} \mathrm{H}$ NMR (DMSO-d $\left.6 . \delta \mathrm{ppm}, 400 \mathrm{MHz}\right): 4.26$ $\left(\mathrm{t}, 1 \mathrm{H}, \mathrm{N}-\mathrm{CH}-\mathrm{CH}_{2}-\right), 3.06\left(\mathrm{~d}, 2 \mathrm{H},-\mathrm{CH}_{2}-\mathrm{Ph}\right), 8.00$ (s, $1 \mathrm{H}$, $\mathrm{NH}), 7.12-7.80$ (m, 9H, aromatic); ${ }^{13} \mathrm{C} \mathrm{NMR}$ (DMSO, $\delta$ ppm): 185.8 (COO), 66.7 (-CH-), $38.3\left(-\mathrm{CH}_{2}-\right), 15.9(\mathrm{C}=\mathrm{N}), 166.4$ $(\mathrm{C}=\mathrm{O}), 149.6,135.7,133.1,128.5,127.6,126.3,124.5,122.7,120.8$ (aromatic carbons); UV-visible $(\lambda \max , \mathrm{nm}): 220,260,320$, 370; infrared $\left(\mathrm{KBr}, \mathrm{cm}^{-1}\right): v(\mathrm{C}=\mathrm{N}), 1609 ; v(\mathrm{C}=\mathrm{O}), 1725$; $v_{\text {asym }}(\mathrm{COO}), 1585 ; v_{\text {sym }}(\mathrm{COO}), 1320 ; v(\mathrm{Zr} \leftarrow \mathrm{N}), 540 ; v(\mathrm{Zr}-$ O), 460.

Compound $\mathrm{Zr}\left(\mathrm{L}^{8}\right)_{2} \mathrm{Cl}_{2}$ was prepared by reacting zirconium(IV) dichloride with ligand $\left(\mathrm{L}^{8} \mathrm{H}\right)$; colour, dark brown; yield, $0.127 \mathrm{~g} ; \mathrm{mp}, 218^{\circ} \mathrm{C}$ (d) and elemental analysis (\%), calcd. for $\mathrm{C}_{38} \mathrm{H}_{28} \mathrm{Cl}_{2} \mathrm{~N}_{6} \mathrm{O}_{6} \mathrm{Zr}$ : C, 55.20; H, 3.41; N, 10.16; found, $\mathrm{C}$ : 55.03; H, 3.36; N, 10.21; molecular weight: found, 821.15, calcd. 826.80. Molar conductance (DMF, $10^{-3}, \Omega^{-1}, \mathrm{moL}^{-1}, \mathrm{~cm}^{2}$ ): $12.4 ;{ }^{1} \mathrm{H}$ NMR (DMSO-d 6 , $\delta$ ppm, $\left.400 \mathrm{MHz}\right): 4.36(\mathrm{t}, 1 \mathrm{H}, \mathrm{N}-$ $\mathrm{CH}-\mathrm{CH}_{2}-$ ); 3.22 (d, 2H, $-\mathrm{CH}_{2}-$ ); 8.06 (s, 1H, NH), 6.98$7.76(\mathrm{~m}, 9 \mathrm{H}$, aromatic); UV-visible $(\lambda \max , \mathrm{nm}): 220,260$, 320, 370; infrared $\left(\mathrm{KBr}, \mathrm{cm}^{-1}\right): v(\mathrm{C}=\mathrm{N}), 1614 ; v(\mathrm{C}=\mathrm{O}), 1726$; $v_{\text {asym }}(\mathrm{COO}), 1594 ; v_{\text {sym }}(\mathrm{COO}), 1318 ; v(\mathrm{Zr} \leftarrow \mathrm{N}), 535 ; v(\mathrm{Zr}-$ $\mathrm{O}), 470$.

\section{Results and Discussion}

The reactions of zirconium(IV) dichloride with the monofunctional bidentate Schiff bases were carried out in dry methanol in 1:2 stoichiometric ratios and can be represented by the equation in Scheme 1.

All these compounds are coloured solids, insoluble in common organic solvents and soluble in DMSO, DMF, $\mathrm{CHCl}_{3}, \mathrm{MeOH}$, and so forth and insoluble in $\mathrm{n}$-hexane and petroleum ether. The conductivity values for the $\mathrm{Zr}(\mathrm{L})_{2} \mathrm{Cl}_{2}$ complexes (in DMF, $10^{-3} \mathrm{moL}, 25^{\circ} \mathrm{C}$ ), ranging in the $8.5-$ $12.6 \Omega^{-1}, \mathrm{moL}^{-1}, \mathrm{~cm}^{2}$ region, indicate that the nonelectrolytic nature of the compounds suggests that the anions are covalently bonded. The molecular weight determination by the Rast camphor method shows that the products are monomeric in nature.

3.1. IR Spectra. The IR absorption frequencies for the ligands and their zirconium complexes were recorded in the range $4000-400 \mathrm{~cm}^{-1}$. The assignment of important infrared data for ligand and complexes are listed in experimental section. 
<smiles>[R]c1ccc2c(c1)C(=O)C(=O)N2</smiles><smiles>[R]C(N)C(=O)O</smiles><smiles>[R]c1ccc2c(c1)/C(=N/C([R])C(=O)O)C(=O)N2</smiles>

$\mathrm{L}^{1} \mathrm{H}-\mathrm{L}^{8} \mathrm{H}$

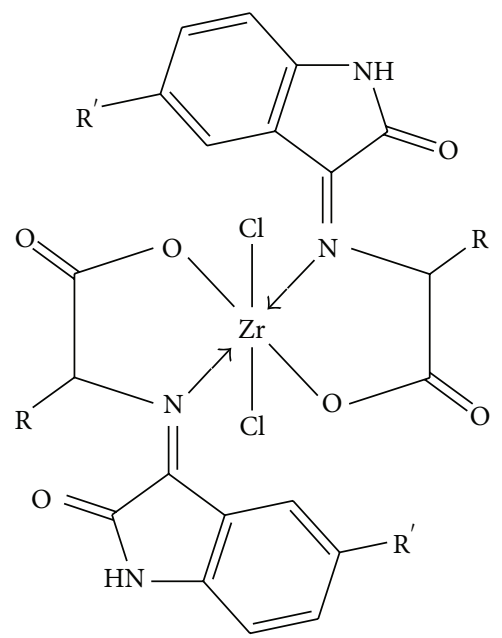

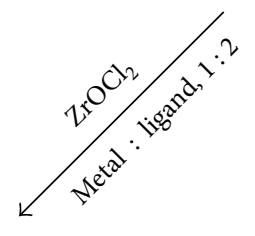

\begin{tabular}{r|lll|ll} 
Abbr. & $\mathrm{R}$ & $\mathrm{R}^{\prime}$ & $\mathrm{Abbr}$ & $\mathrm{R}$ & $\mathrm{R}$ \\
\hline $\mathrm{L}^{1} \mathrm{H}$ & $-\mathrm{H}$ & $\mathrm{H}$ & $\mathrm{L}^{2} \mathrm{H}$ & $-\mathrm{CH}_{3}$ & $\mathrm{H}$ \\
$\mathrm{L}^{3} \mathrm{H}$ & $-\mathrm{CH}\left(\mathrm{CH}_{3}\right)_{2}$ & $\mathrm{H}$ & $\mathrm{L}^{4} \mathrm{H}$ & $-\left(\mathrm{CH}_{2}\right)_{2} \mathrm{SCH}_{3}$ & $\mathrm{H}$ \\
$\mathrm{L}^{5} \mathrm{H}$ & $-\mathrm{CH}_{3}$ & $\mathrm{Cl}$ & $\mathrm{L}^{6} \mathrm{H}$ & $-\mathrm{Cl}$
\end{tabular}

Scheme 1: Representative equation illustrating the formation of Schiff bases and their zirconium(IV) complexes.

A strong band (due to the azomethine group) at 1620$1635 \mathrm{~cm}^{-1}$ in the spectra of the free ligands $[27,28]$ is shifted to lower wavenumber in the complexes studied, showing the coordination of azomethine nitrogen to the metal atom [26, 29]. The coordination of azomethine nitrogen to the metal atom is supported by the appearance of a new absorption band at $535 \mathrm{~cm}^{-1}$, which may be assigned to $v(\mathrm{Zr} \leftarrow \mathrm{N})$ vibrations [30].

The infrared spectra of all the zirconium complexes do not show the strong band in the region $3105-2740 \mathrm{~cm}^{-1}$ due to $v(\mathrm{COOH})$, indicating the deprotonation of the carboxylate group of the Schiff bases with zirconium metal as expected. It is further confirmed by the appearance of sharp band at $465 \mathrm{~cm}^{-1}$ in the spectra of all the complexes assignable to the $v(\mathrm{Zr}-\mathrm{O})$ stretching vibrations [31]. In the spectra of the complexes two sharp bands are observed at 1590 and $1320 \mathrm{~cm}^{-1}$ and are assigned to the $v_{\text {asym }}$ (COO) and $v_{\text {sym }}(\mathrm{COO})$, respectively. Furthermore, the separation between asymmetric and symmetric vibrations is about $270 \mathrm{~cm}^{-1}$, indicating the covalent nature of the metal-oxygen 


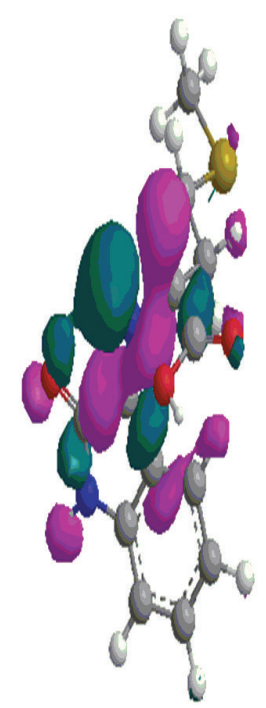

(a)

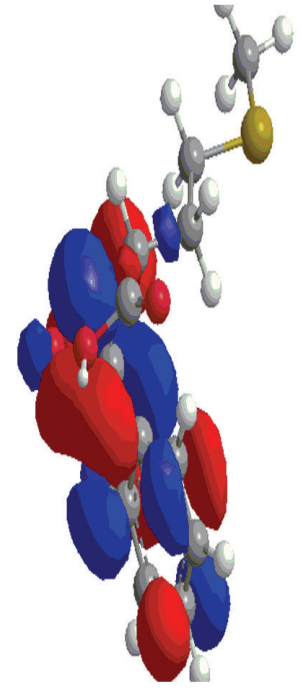

(b)

FIGURE 3: (a) HOMO orbitals of the MM2 geometry-optimized structure of the ligand $\left(\mathrm{L}^{4} \mathrm{H}\right)$. (b) LUMO orbitals of the MM2 geometryoptimized structure of the ligand $\left(\mathrm{L}^{4} \mathrm{H}\right)$.

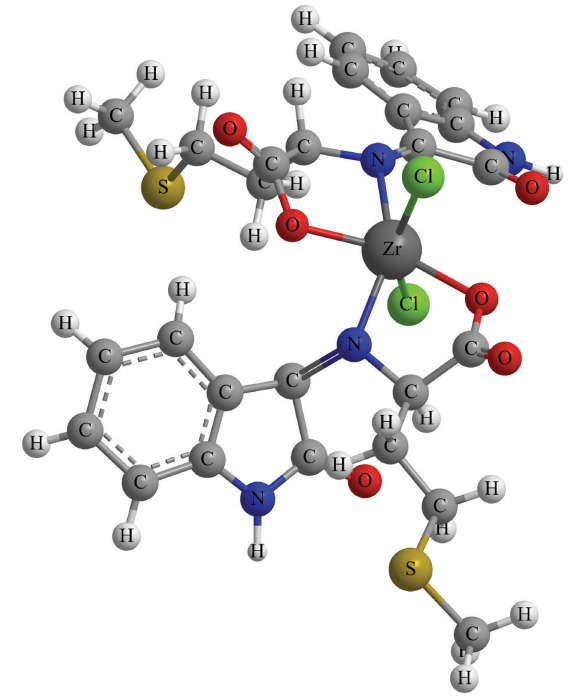

Figure 4: 3D molecular structure of $\mathrm{Zr}\left(\mathrm{L}^{4}\right)_{2} \mathrm{Cl}_{2}$.

bond as unidentate manner. Ionic bonding and also bridging or chelation can therefore be excluded. Sandhu and Verma in their studies and reports have shown that the $\Delta v$ value of complexes greater by $65-90 \mathrm{~cm}^{-1}$ than in their sodium salts indicates either asymmetric or monodentate bonding of the carboxylate group to metal atom [32]. Moreover, $\Delta v$ values of complexes below $200 \mathrm{~cm}^{-1}$ would be expected for bridging or chelating carboxylates, but greater than $200 \mathrm{~cm}^{-1}$ for the monodentate bonding carboxylate anions [33]. The $\mathrm{C}=\mathrm{O}$ band of the indole group appears in the range of 1720 $1740 \mathrm{~cm}^{-1}$ in the ligands. However, a strong band at $1740 \mathrm{~cm}^{-1}$ due to the vibration of $\mathrm{C}=\mathrm{O}$ group remains unchanged in the spectra of complexes showing thereby the noninvolvement of this group in coordination and thus confirms that the $\mathrm{C}=\mathrm{O}$ from indole is not involved in the complexation.

3.2. Electronic Spectra. The electronic spectra of Schiff bases and its zirconium complexes have been recorded in methanol. In spectrum of the ligand, three bands are observed at 280 , 300 , and $380 \mathrm{~nm}$. The bands at 280 and $300 \mathrm{~nm}$ are due to the $\pi-\pi^{*}$ transitions within the aromatic ring and remain almost unchanged in the spectra of zirconium complexes. Another band at $380 \mathrm{~nm}$ is due to the $n-\pi^{*}$ transitions within the $>\mathrm{C}=\mathrm{N}-$ chromophore and shows a bathochromic shift in the spectra of zirconium complexes due to the coordination of azomethine nitrogen to the zirconium atom. This band shifts slightly to the higher-energy region in the spectra of zirconium complexes due to the polarization within the $>\mathrm{C}=\mathrm{N}$ chromophore caused by the zirconium-ligand electron interaction.

3.3. ${ }^{1} H$ NMR Spectra. The characteristic resonance peaks for the synthesized compounds have been recorded in DMSO$\mathrm{d}_{6}$ and data are given in experimental section. The expected resonances are assigned by their peak multiplicity, intensity patterns, and integration. The ${ }^{1} \mathrm{H}$ NMR spectral data of the ligands show single resonance at $\delta 11.4 \pm 0.10 \mathrm{ppm}$, which is absent in the spectra of the metal complexes, indicating the replacement of the carboxylic acid proton by the $\mathrm{Zr}(\mathrm{IV})$ moiety. The ligands give a complex multiplet signal in the region $\delta 7.20-7.82 \mathrm{ppm}$ for the aromatic protons and these remain almost at the same position in the spectra of the metal complexes. The appearance of signals due to $\mathrm{NH}$ protons at the same positions in the ligand and its complexes shows the non-involvement of this group in coordination.

Schiff bases derived from glycine, alanine, valine, and methionine display four/three aromatic protons, as expected. In the spectrum of phenylalanine, the integral of the aromatic 
region corresponds to nine protons; five protons on the phenyl ring are recognizable at $7.4 \mathrm{ppm}$. Methylene (for glycine, alanine, valine, and methionine) protons on the $\alpha$-carbon of the carboxylic acid moieties appear at 3.96$4.30 \mathrm{ppm}$. This signal is a singlet for (1), a doublet for (3) and (6), a triplet for (7) and (8), and a quartet for (2) and (5) all of which arise from the nonequivalent methylene protons in structures (1-8). In general, the complexes obtained were found to exhibit no additional resonances and thus reflect the purity of the complexes. The integration of peaks concurs with the number of protons postulated from the structures proposed for the complexes.

3.4. ${ }^{13} \mathrm{C} N M R$ Spectra. The ${ }^{13} \mathrm{C}$ NMR spectral data for ligands and their corresponding metal complexes have been recorded in experimental part. Evidence of the formation of the complexes is clearly displayed in the ${ }^{13} \mathrm{C} \mathrm{NMR}$ spectra. The ${ }^{13} \mathrm{C}$ NMR spectra of complexes showed that the $\delta(\mathrm{COO})$ signal shifted to the downfield region which is lower compared to that of the ligand (176.1-178.5 ppm) indicating the carboxylate anion is bonded to zirconium atom upon complexation. The signals due to the carbon atom attached to the azomethine group in the ligands appear at $\delta 162.3 \pm$ $0.90 \mathrm{ppm}$. However, in the spectra of the corresponding metal complexes, these appear at $\sim \delta 154.2 \mathrm{ppm}$. The considerable shifts in the resonance of the carbon atom attached to nitrogen indicate the involvement of azomethine nitrogen atom of coordination. The occurrence of eight resonances in the range of $\delta 118.3-150.7 \mathrm{ppm}$ in the ${ }^{13} \mathrm{C} \mathrm{NMR}$ spectra of the complexes and ligands is defined as aromatic carbons signals. Generally, the ${ }^{13} \mathrm{C}$ NMR spectra of the complexes obtained were found to exhibit no additional resonances and thus reflected the purity of the complexes.

Though, it is also possible that the shifting of azomethine carbon is due to the change in hybridization of nitrogen attached to carboxylate group, but in the light of IR, UV, and ${ }^{1} \mathrm{H}$ NMR spectral studies it seems more plausible that the shifting in these carbons is due to the involvement of carboxylate oxygen and azomethine nitrogen in bonding.

3.5. Fluorescence Spectral Studies. Metal-ligand coordination may lead to significant changes of the fluorescence properties of the ligand, including increase of the intensity, shift of the emission wavelength, appearance of new emissions, or quenching of the fluorescence. The fluorescence spectra of the Schiff base $\mathrm{HL}^{1}$ and $\mathrm{HL}^{3}$ and their metal complexes were recorded in DMF with excitation wavelength $380 \mathrm{~nm}$ at room temperature $(298 \mathrm{~K})$. The fluorescence emission spectrum of $\mathrm{HL}^{1}$ with its metal complexes is depicted in Figures 1 and 2. $\mathrm{HL}^{1}$ exhibits a strong fluorescence emission at $430 \mathrm{~nm}$; in contrast with this partial fluorescence quenching phenomena are observed in its metal complexes with weak fluorescence emission at $432 \mathrm{~nm}$ for zirconium(IV). $\mathrm{HL}^{3}$ shows strong fluorescence band at $435 \mathrm{~nm}$ and its $\mathrm{Zr}$ (IV) complexes exhibit weak emission bands at $436 \mathrm{~nm}$, respectively. The maximum emission wavelength of Schiff bases is red-shifted about 10$20 \mathrm{~nm}$ owing to the formation of complex, which may be tentatively assigned to the ligand to metal charge transfer
(LMCT). It is evident from the fluorescence spectra that fluorescence emission intensity of Schiff bases decreased dramatically on complex formation with transition metal ions. The decrease in fluorescence intensity with formation of metal complexes is due to decrease in electron density on Schiff bases [34, 35]. All these fluorescent emissions may be assigned to the intraligand fluorescence since the free ligand exhibited a similar emission at 430 and $435 \mathrm{~nm}$, respectively, under the same condition.

3.6. Molecular Modeling and Analysis. The ligand $\left(\mathrm{L}^{4} \mathrm{H}\right)$ has 50 filled molecular orbitals, and the orbitals of the HOMO and LUMO levels are shown in Figures 3(a) and 3(b). Notice that the HOMO orbitals are concentrated on the oxygen and nitrogen atoms while the LUMO orbitals are concentrated on the carbons of the indole rings. The HOMO and LUMO energy was found to be $-8.268 \mathrm{eV}$ and $-4.368 \mathrm{eV}$.All the synthesized compounds have hexa-coordination and distorted octahedral geometry. Molecular modeling was performed for $\left[\mathrm{ZrCl}_{2}\left(\mathrm{~L}^{4}\right)_{2}\right]$, as representative compound. Tables 1 and 2 list selected interatomic distances and bond angles. The structure of the complex with atomic numbering scheme is shown in Figure 4. The complex consists of two units of ligand molecule with metal ion $\mathrm{Zr}(\mathrm{IV})$. The complex is of six coordinates with distorted octahedral environment around the metal ion. The metal ion $\mathrm{Zr}(\mathrm{IV})$ is coordinated to one azomethine nitrogen atom and one carboxylate oxygen atom of Schiff-base ligand and $\mathrm{Cl}$ ion. The $\mathrm{Zr}-\mathrm{O}$ and $\mathrm{Zr}-\mathrm{N}$ bond lengths are $2.092 \AA$ and $2.138 \AA$, respectively, and the bond distance between $\mathrm{Zr}-\mathrm{Cl}$ is $2.444 \AA$. The two chloride groups are equidistant from $\mathrm{Zr}(2.44 \AA)$ and complete the coordination sphere of $\mathrm{Zr}$. The $\mathrm{Cl}-\mathrm{Zr}-\mathrm{Cl}$ angles are $154.92^{\circ}$. The $\mathrm{O}-\mathrm{Zr}-\mathrm{N}, \mathrm{N}-\mathrm{Zr}-\mathrm{N}$, and $\mathrm{O}-\mathrm{Zr}-\mathrm{O}$ angles are 80.11, 149.08, and $148.23^{\circ}$, respectively, for both the Schiff-base ligands. These angles and distances are in good agreement with the $\mathrm{X}$-ray structure of a similar zirconium complex [36]. The $\mathrm{Zr}-$ $\mathrm{Cl}$ distances for the metal bound chloride groups are also similar in both complexes and are comparable with reported zirconium compounds [37].

In all, 205 measurements of the bond lengths (72 in numbers) plus the bond angles (133 in numbers) are listed. Except for a few cases, optimum values of both the bond lengths and the bond angles are given in the tables, with the actual values. The actual bond lengths/bond angles given in Tables 1 and 2 are calculated values as a result of energy optimization in Chem3D Ultra, and the optimum bond length/bond angle values are the standard bond lengths/bond angles established by the builder unit of CHEM 3D. Some values of standard bond lengths/bond angles are missing, possibly because of limitations of the software; we have already noticed this when modeling other systems. In most cases, the actual bond lengths and bond angles are close to the optimum values, confirming the proposed structure of the compound $\left[\mathrm{Zr}\left(\mathrm{L}^{4}\right)_{2} \mathrm{Cl}\right]$.

\section{Conclusion}

New Schiff bases and their zirconium complexes have been successfully synthesized. Elemental analysis data obtained 
are in good agreement with the predicted formula. Distorted octahedral geometries have been proposed for $\mathrm{Zr}$ (IV) complexes with the help of various physicochemical studies. The infrared spectra of these complexes show the presence of monofunctional and bidentate ligands. The Schiff base exhibits a strong fluorescence emission; in contrast with this partial fluorescence quenching phenomena is observed in its metal complexes. The proposed structures of metal complexes are presented in Figure 4 . The ${ }^{1} \mathrm{H}$ NMR spectra showed that the calculated number of protons for each functional group in the complexes is equal to the number predicted from the molecular formula. Moreover, the ${ }^{1} \mathrm{H}$ and ${ }^{13} \mathrm{C}$ NMR spectra of the complexes obtained were found to exhibit no additional resonances and thus reflected the purity of the metal complexes.

\section{Acknowledgments}

The authors are thankful to the Dean, Faculty of Engineering \& Technology, Mody Institute of Technology and Science, Deemed University, Lakshmangarh, Sikar, for providing necessary facilities and financial support. They are also thankful to the Head, SAIF, Panjab University, Chandigarh, for providing elemental analysis and NMR facilities. The authors are also grateful to Dr. Sangeeta Jhajharia for linguistic corrections.

\section{References}

[1] G. Cerchiaro, K. Aquilano, G. Filomeni, G. Rotilio, M. R. Ciriolo, and A. M. D. C. Ferreira, "Isatin-Schiff base copper(II) complexes and their influence on cellular viability," Journal of Inorganic Biochemistry, vol. 99, no. 7, pp. 1433-1440, 2005.

[2] A. J. Abdul-Ghani and A. M. N. Khaleel, "Synthesis and characterization of new Schiff bases derived from N, (1)substituted isatin with dithiooxamide and their $\mathrm{Co}(\mathrm{II}), \mathrm{Ni}(\mathrm{II})$, $\mathrm{Cu}(\mathrm{II}), \mathrm{Pd}(\mathrm{II})$, and $\mathrm{Pt}(\mathrm{IV})$ complexes," Bioinorganic Chemistry and Applications, vol. 2009, Article ID 413175, 12 pages, 2009.

[3] R. A. Kusanur, M. Ghate, and M. V. Kulkarni, "Synthesis of spiro[indolo-1,5-benzodiazepines] from 3-acetyl coumarins for use as possible antianxiety agents," Journal of Chemical Sciences, vol. 116, no. 5, pp. 265-270, 2004.

[4] A. Ercag, S. O. Yildirim, M. Akkurt, M. U. Ozgur, and F. W. Heinemann, "Novel isatin-Schiff base $\mathrm{Cu}(\mathrm{II})$ and $\mathrm{Ni}(\mathrm{II})$ complexes. X-ray crystal structure of bis [3-(4-hexylphenylimino)-1H-indol-2(3H)-one]-dichlorocopper(II) complex," Chinese Chemical Letters, vol. 17, no. 2, pp. 243-246, 2006.

[5] F. Lu, R. A. Zarkesh, and A. F. Heyduk, "A redox-active ligand as a reservoir for protons and electrons: $\mathrm{O}_{2}$ reduction at zirconium(IV)," European Journal of Inorganic Chemistry, vol. 2012, no. 3, pp. 467-470, 2012.

[6] L. Kakaliou, W. J. Scanlon, B. Qian, S. W. Baek, M. R. Smith, and D. H. Motry, "Five- and six-coordinate group 4 compounds stabilized by $\beta$-ketiminate and diketiminate ligands: syntheses and comparisons between solid-state and solution structures," Inorganic Chemistry, vol. 38, no. 26, pp. 5964-5977, 1999.

[7] M. Coluccia, A. Nassi, A. Boccarelli et al., "In vitro and in vivo antitumour activity and cellular pharmacological properties of new platinum-iminoether complexes with different configuration at the iminoether ligands," Journal of Inorganic Biochemistry, vol. 77, no. 1-2, pp. 31-35, 1999.

[8] C. T. Supuran, F. Mincione, A. Scozzafava, F. Briganti, G. Mincione, and M. A. Ilies, "Carbonic anhydrase inhibitorspart 52. Metal complexes of heterocyclic sulfonamides: a new class of strong topical intraocular pressure-lowering agents in rabbits," European Journal of Medicinal Chemistry, vol. 33, no. 4, pp. 247-254, 1998.

[9] H. L. Singh and J. Singh, "Synthesis, spectral, 3D molecular modeling and antibacterial studies of dibutyltin (IV) Schiff base complexes derived from substituted isatin and amino acids," Natural Science, vol. 4, no. 3, pp. 170-178, 2012.

[10] D. Singh, A. Kumari, R. V. Singh, S. M. Mehta, I. J. Gupta, and K. Singh, "Antifertility and biocidal activities of organometallics of silicon, germanium, titanium and zirconium derived from 2-acetylthiophene thiosemicarbazone," Applied Organometallic Chemistry, vol. 7, no. 4, pp. 289-292, 1993.

[11] M. P. Sathisha, V. K. Revankar, and K. S. R. Pai, "Synthesis, structure, electrochemistry, and spectral characterization of bisisatin thiocarbohydrazone metal complexes and their antitumor activity against ehrlich ascites carcinoma in Swiss Albino mice," Metal-Based Drugs, vol. 2008, Article ID 362105, 11 pages, 2008.

[12] B. Rosenberg and L. VanCamp, "The successful regression of large solid sarcoma 180 tumors by platinum compounds," Cancer Research, vol. 30, no. 6, pp. 1799-1802, 1970.

[13] N. Dharmaraj, P. Viswanathamurthi, and K. Natarajan, "Ruthenium(II) complexes containing bidentate Schiff bases and their antifungal activity," Transition Metal Chemistry, vol. 26, no. 1-2, pp. 105-109, 2001.

[14] A. S. El-Tabl, M. M. E. Shakdofa, A. M. A. El-Seidy, and A. N. Al-Hakimi, "Synthesis, characterization and antifungal activity of metal complexes of 2-(5-((2-chlorophenyl)diazenyl)-2hydroxybenzylidene) hydrazinecarbothioamide," Phosphorus, Sulfur, and Silicon and the Related Elements, vol. 187, no. 11, pp. 1312-1323, 2012.

[15] H. L. Singh, "Synthesis and characterization of tin(II) complexes of fluorinated Schiff bases derived from amino acids," Spectrochimica Acta A, vol. 76, no. 2, pp. 253-258, 2010.

[16] T. Jeewoth, H. L. K. Wah, M. G. Bhowon, D. Ghoorohoo, and K. Babooram, "Synthesis and anti-bacterial/catalytic properties of Schiff bases and Schiff base metal complexes derived from 2,3-diaminopyridine," Synthesis and Reactivity in Inorganic and Metal-Organic Chemistry, vol. 30, no. 6, pp. 1023-1038, 2000.

[17] S. N. Pandeya, A. S. Raja, and J. P. Stables, "Synthesis of isatin semicarbazones as novel anticonvulsants-role of hydrogen bonding," Journal of Pharmacy and Pharmaceutical Sciences, vol. 5, no. 3, pp. 266-271, 2002.

[18] S. P. Singh, S. K. Shukla, and L. P. Awasthi, "Synthesis of some3( 4 '-nitro-benzoylhydrazone)-2-Indolinones as potential antiviral agents," Current Science, vol. 52, no. 16, pp. 766-769, 1983.

[19] J. Strauch, T. H. Warren, G. Erker, R. Fröhlich, and P. Saarenketo, "Formation and structural properties of salicylaldiminato complexes of zirconium and titanium," Inorganica Chimica Acta, vol. 300-302, pp. 810-821, 2000.

[20] P. G. Cozzi, "Metal-Salen Schiff base complexes in catalysis: practical aspects," Chemical Society Reviews, vol. 33, no. 7, pp. 410-421, 2004.

[21] C. K. Modi, S. H. Patel, and M. N. Patel, "Transition metal complexes with uninegative bidentate Schiff base: synthetic, thermal, spectroscopic and coordination aspects," Journal of 
Thermal Analysis and Calorimetry, vol. 87, no. 2, pp. 441-448, 2007.

[22] C. Spînu, M. Pleniceanu, and C. Tigae, "Biologically active transition metal chelates with a 2-thiophenecarboxaldehydederived Schiff base: synthesis, characterization, and antibacterial properties," Turkish Journal of Chemistry, vol. 32, no. 4, pp. 487-493, 2008.

[23] H. L. Singh and A. K. Varshney, "Synthesis and characterization of coordination compounds of organotin(IV) with nitrogen and sulfur donor ligands," Applied Organometallic Chemistry, vol. 15, no. 9, pp. 762-768, 2001.

[24] K. Sharma, N. Fahmi, and R. V. Singh, "Biologically potent new heterobimetallic complexes of platinum, silicon, tin, titanium and zirconium," Main Group Metal Chemistry, vol. 25, no. 12, pp. 727-732, 2002.

[25] G. Rubner, K. Bensdorf, A. Wellner et al., "Synthesis and biological activities of transition metal complexes based on acetylsalicylic acid as neo-anticancer agents," Journal of Medicinal Chemistry, vol. 53, no. 19, pp. 6889-6898, 2010.

[26] H. L. Singh and J. B. Singh, "Synthesis and characterization of new lead(II) complexes of Schiff bases derived from amino acids," Research on Chemical Intermediates, 2012.

[27] H. L. Singh, J. B. Singh, and K. P. Sharma, "Synthetic, structural, and antimicrobial studies of organotin(IV) complexes of semicarbazone, thiosemicarbazone derived from 4-hydroxy3-methoxybenzaldehyde," Research on Chemical Intermediates, vol. 38, no. 1, pp. 53-65, 2012.

[28] M. Nath, H. Singh, G. Eng, X. Song, and A. Kumar, "Syntheses, characterization and biological activity of diorganotin(IV) derivatives of 2-amino-6-hydroxypurine (guanine)," Inorganic Chemistry Communications, vol. 12, no. 10, pp. 1049-1052, 2009.

[29] B. Khera, A. K. Sharma, and N. K. Kaushik, "Bis(indenyl)titanium(IV) and zirconium(IV) complexes of monofunctional bidentate salicylidimines," Polyhedron, vol. 2, no. 11, pp. 11771180, 1983.

[30] V. Srivastava, S. K. Sengupta, and S. C. Tripathi, "Coordination compounds of zirconium(lV) with thiosemicarbazones," Synthesis and Reactivity in Inorganic and Metal-Organic Chemistry, vol. 15, no. 2, pp. 163-173, 1985.

[31] R. K. Dubey, A. Singh, and R. C. Mehrotra, "Synthesis, reactions, spectral and magnetic studies of bimetallic alkoxides of cobalt(II) with zirconium(IV)," Inorganica Chimica Acta, vol. 118, no. 2, pp. 151-156, 1986.

[32] G. K. Sandhu and S. P. Verma, "Triorganotin(IV) derivatives of five membered heterocyclic 2-carboxylic acids," Polyhedron, vol. 6, no. 3, pp. 587-592, 1987.

[33] H. L. Singh and J. B. Singh, "Synthesis and characterization of new lead(II) and organotin(IV) complexes of Schiff bases derived from histidine and methionine," International Journal of Inorganic Chemistry, vol. 2012, Article ID 568797, 7 pages, 2012.

[34] B. A. Yamgar, V. A. Sawant, S. K. Sawant, and S. S. Chavan, "Copper(II) complexes of thiazolylazo dye with triphenylphosphine and $\mathrm{N}_{3}^{-}$or $\mathrm{NCS}^{-}$as coligands: synthesis, spectral characterization, electrochemistry and luminescence properties," Journal of Coordination Chemistry, vol. 62, no. 14, pp. 2367-2374, 2009.

[35] X. H. Lu, Y. Q. Huang, L. Y. Kong, T. A. Okamura, W. Y. Sun, and N. Ueyama, "Syntheses, structures and luminescent properties of three silver(I) complexes with a novel imidazole-containing schiff base ligand," Zeitschrift fur Anorganische und Allgemeine Chemie, vol. 633, no. 11-12, pp. 2064-2070, 2007.

[36] C. Parnav, A. Kriza, V. Pop, and S. Udrea, "Complexes of tin(IV) and zirconium(IV) with Schiff bases derived from isatin and diamines," Journal of the Indian Chemical Society, vol. 82, no. 1, pp. 71-73, 2005.

[37] D. P. Krut'Ko, M. V. Borzov, E. N. Veksler, A. V. Churakov, and J. A. K. Howard, "(2-diphenylphosphinoethyl)cyclopentadienyl complexes of zirconium (IV): synthesis, crystal structure and dynamic behaviour in solutions," Polyhedron, vol. 17, no. 22, pp. 3889-3901, 1999. 

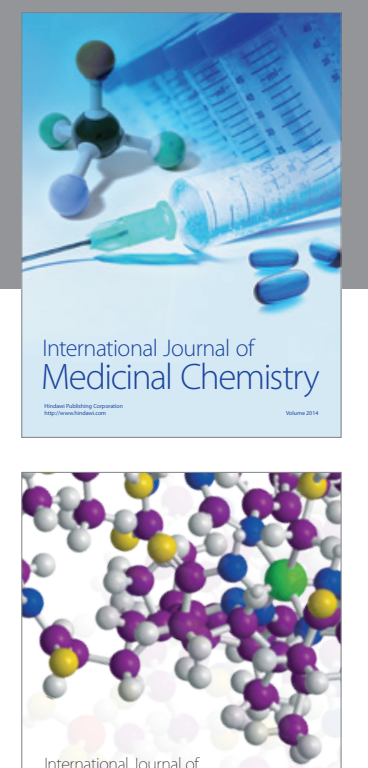

\section{Carbohydrate} Chemistry

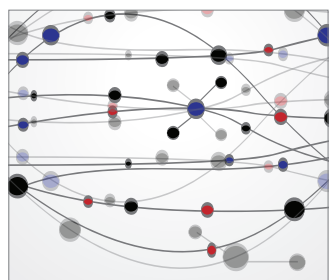

The Scientific World Journal
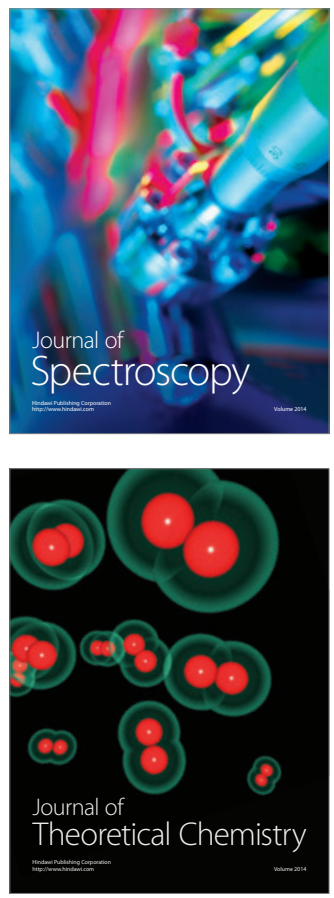
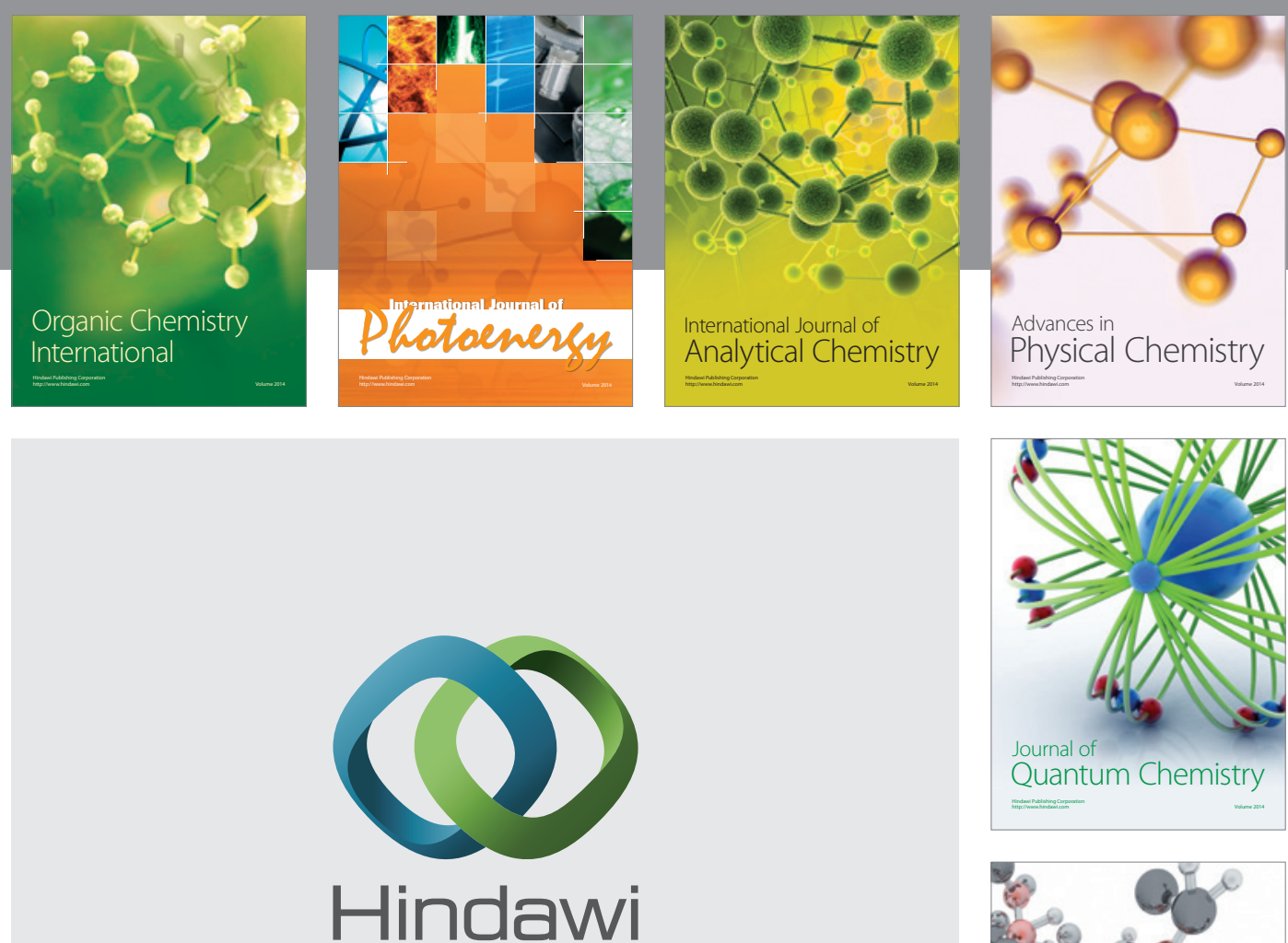

Submit your manuscripts at

http://www.hindawi.com

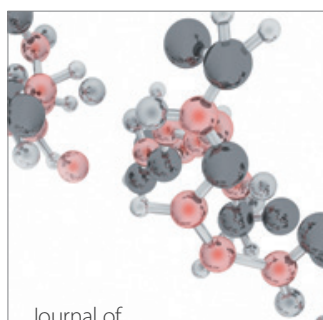

Analytical Methods

in Chemistry

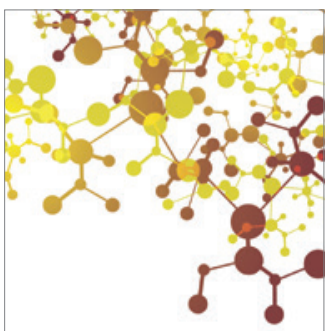

Journal of

Applied Chemistry

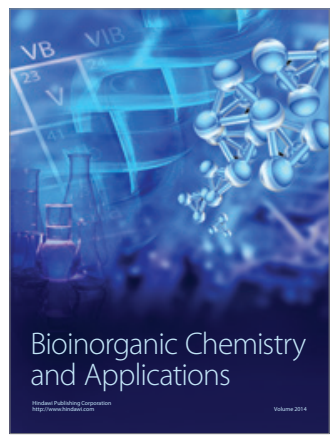

Inorganic Chemistry
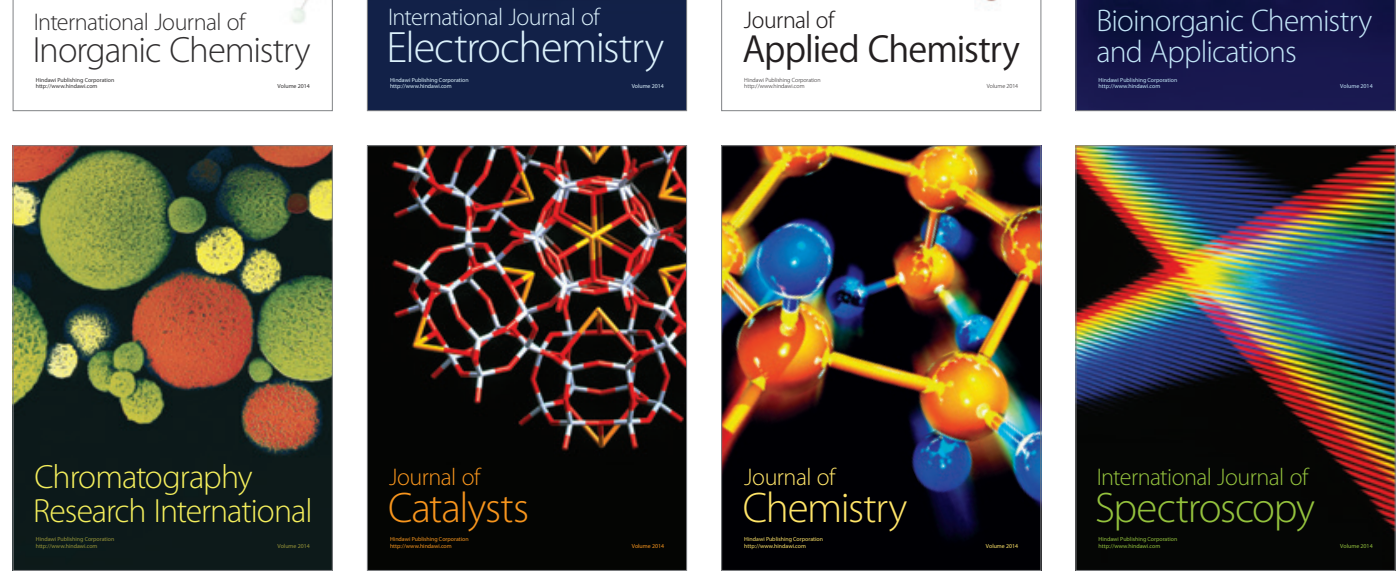